\title{
A POLÍTICA NACIONAL DE MEDICAMENTOS (PNM) SOB A ÓTICA DOS DIREITOS HUMANOS
}

\author{
NATIONAL DRUG POLICY (PNM) UNDER THE VIEW OF HUMAN RIGHTS \\ POLÍTICA NACIONAL DE DROGAS (PNM) BAJO LA VISIÓN DE LOS \\ DERECHOS HUMANOS
}

Guilherme Lucas Pinheiro ${ }^{1}$

\author{
RECEIVED /RECEBIDO 25/07/2019 MAI 292019 \\ APPROVED/APROVADO 29/07/2019 JUNE 302019 \\ PUBLISHED /PUBLICADO 05/08/2019 JULY 072019 \\ Editor Responsável: Carla Caldas \\ Método de Avaliação: Double Blind Review \\ E-ISSN: 2316-8080 \\ Prefixo do DOI: 10.16928
}

\section{RESUMO}

O objetivo deste estudo foi identificar se há simetria entre as ações da Política Nacional de Medicamentos com as diretrizes de Direitos Humanos. Utilizou-se o estudo transversal, com levantamento de dados entre os anos 2008 a 2017, que considerou as variáveis demográficas, sociais, econômicas, dispensação de medicamentos, e que teve como fontes a Política Nacional de Medicamentos, a Política Nacional de Assistência Farmacêutica, a Pesquisa Nacional por Amostra de Domicílios de 2008, do Instituto Brasileiro de Geografia e Estatísticas e a Pesquisa Nacional de Acesso, Utilização e Promoção do Uso Racional de Medicamentos. Em relação aos resultados, destacou-se o Programa Farmácia Popular do Brasil criado em 2004 que atendeu cerca de 47.416.735 usuários até o final de 2017, ou seja, aproximadamente $23 \%$ da população brasileira teve acesso aos medicamentos que necessitavam por consequência da implementação desse programa. Assim, concluiu-se que a Política Nacional de Medicamentos vem ao longo dos anos melhorando o acesso aos medicamentos, uma vez que a Relação Nacional de Medicamentos Essenciais (RENAME) é atualizada a cada dois anos, alinhando-se com as diretrizes da Organização Mundial da Saúde.

Palavras-chave: Acesso à Medicamentos. Assistência Farmacêutica. Uso Racional de Medicamentos. Medicamentos Essenciais. Direitos Humanos.

\begin{abstract}
The aim of this study was to identify if there is symmetry between the actions of the National Medicines Policy and the Human Rights guidelines. We used the cross-sectional study, with data collection from 2008 to 2017, which considered the demographic, social, economic variables, drug dispensing, and which had as sources the National Policy of Medicines, the National Policy of Pharmaceutical Assistance, the 2008 National Household Sample Survey

\footnotetext{
${ }^{1}$ É advogado e professor universitário. Possui especialização em Direito Processual pela Pontifícia Universidade Católica de Minas Gerais (2018), pós-graduando em Direito Empresarial pela Escola Paulista de Direito e mestrando em Sociedade, Tecnologias e Políticas Públicas pelo Centro Universitário Tiradentes (UNIT-AL). ORCID: https://orcid.org/0000-0001-6084-6639. E-mail: guilhermelucaspinheiro@outlook.com.
}

PIDCC, Aracaju/Se, Ano VIII, Volume 13 no 03, p.093 a 105 Out/2019 | www.pidcc.com.br 
of the Brazilian Institute of Geography and Statistics; and the National Survey on Access, Use and Promotion of Rational Use of Medicines. Regarding the results, we highlight the Popular Pharmacy Program of Brazil created in 2004 that served about 47,416,735 users by the end of 2017, ie approximately $23 \%$ of the Brazilian population had access to the medicines they needed as a result of the implementation. of this program. Thus, it was concluded that the National Medicines Policy has been improving access to medicines over the years, as the National List of Essential Medicines (RENAME) is updated every two years, in line with the World Organization guidelines. of health.

Keywords: Access to Medications. Pharmaceutical care. Rational Use of Medications. Essential Medicines. Human rights.

\section{RESUMEN}

El objetivo de este estudio fue identificar si existe una simetría entre las acciones de la Política Nacional de Medicamentos y las directrices de Derechos Humanos. Utilizamos el estudio transversal, con recopilación de datos de 2008 a 2017, que consideró las variables demográficas, sociales, económicas, la dispensación de medicamentos y que tuvo como fuentes la Política Nacional de Medicamentos, la Política Nacional de Asistencia Farmacéutica, la Encuesta Nacional de Muestra de Hogares 2008 del Instituto Brasileño de Geografía y Estadística, y la Encuesta Nacional sobre Acceso, Uso y Promoción del Uso Racional de Medicamentos. En cuanto a los resultados, destacamos el Programa de Farmacia Popular de Brasil creado en 2004 que atendió a unos 47,416,735 usuarios a fines de 2017, es decir, aproximadamente el $23 \%$ de la población brasileña tenía acceso a los medicamentos que necesitaba como resultado de la implementación. de este programa. Por lo tanto, se concluyó que la Política Nacional de Medicamentos ha mejorado el acceso a los medicamentos a lo largo de los años, ya que la Lista Nacional de Medicamentos Esenciales (RENAME) se actualiza cada dos años, de acuerdo con las directrices de la Organización Mundial. Salud.

Palabras clave: Acceso la drogas. Asistencia Farmacéutica. Uso racional de drogas. Drogas Esenciales. Derechos humanos.

\section{INTRODUÇÃO}

A atenção sobre políticas públicas voltadas aos medicamentos no Brasil surgiu por meio da Lei 8.080 de 1990 que criou o Sistema Único de Saúde (SUS), com a finalidade de garantir o acesso integral, universal, igualitário e gratuito aos serviços de saúde, e trouxe a formulação da política de medicamentos como um de seus objetivos (BERTOLDI et al., 2016, p. 2).

Dando sequência, no ano de 1993 foi publicado o Decreto no 793 pela Presidência da República, buscando favorecer a redução de preços dos medicamentos e destacou a denominação genérico. Esse Decreto foi revogado em 1999 por meio do Decreto no 3.181 que 
ratificou as políticas voltadas aos medicamentos, ao estabelecer e dispor sobre medicamentos genéricos (BRASIL, 1999).

Ademais, a Política Nacional de Medicamentos (PNM) instituída pela Portaria $\mathrm{n}^{\mathrm{o}}$ 3.916 de 1998, do Ministério da Saúde, é um marco para a efetivação de ações capazes de melhorar a assistência em saúde da população, pois visa "a garantia da segurança, eficácia e qualidade dos medicamentos, a promoção do uso racional e o acesso da população àqueles considerados essenciais" (VASCONCELOS et al., 2017, p. 2610).

A denominação medicamento genérico é definida pela Lei no 9.787 de 1999 (que alterou a Lei 6.360 de 1976 que trata da denominação de fármacos) como medicamento similar a um produto de referência ou inovador, desenvolvido quando a proteção patentária foi afastada, seja pela expiração do prazo ou pela renúncia do proprietário da patente em desenvolver o medicamento (BRASIL, 1999).

Posto isto, importante mencionar os dados da Pesquisa Nacional de Saúde (PNS) 2013, onde 41,5\% da população urbana e rural brasileira com 18 anos ou mais foi diagnosticada com hipertensão arterial, 11,1\% com diabetes, 7,4\% com alguma doença do coração, 13,6\% com depressão e etc. Estas informações são validadas quando analisadas sob a ótica da lista de medicamentos disponibilizados pela ação "aqui tem farmácia popular", onde os medicamentos são vendidos ao consumidor por preços diferenciados.

A Política Nacional de Medicamentos (PNM) vem ao longo dos anos, por meio da Relação Nacional de Medicamentos Essenciais (Renama) melhorando o acesso aos medicamentos, uma vez que a cada dois anos, esta relação é atualizada, alinhando-se com as diretrizes da Organização Mundial da Saúde (OMS).

A Rename 2018 contém 878 medicamentos, sendo que 11 desses foram incluídos como novas opções para tratamento de diferentes doenças, entre elas: a mucopolissacaridose tipo I, mucopolissacaridose tipo II, polineuropatia amiloidótica familiar relacionada à proteína transtirretina e doença de Wilson. Além disso, dois fármacos: o adalimumabe e o everolimo tiveram ampliação de uso. Já o artemeter ( $80 \mathrm{mg} / \mathrm{mL}$, solução injetável) (CONITEC, 2018).

\section{MÉTODOS}

Visando alcançar o objetivo de identificar se há simetria entre as ações da Política Nacional de Medicamentos (PNM) com as diretrizes de Direitos Humanos, utilizou-se um 
estudo transversal com abordagem quali-quantitativa. O estudo fez um levantamento de dados entre os anos 2008 a 2017, e considerou as variáveis demográficas, sociais, econômicas, dispensação de medicamentos e preços no setor privado. E teve como fontes a Política Nacional de Medicamentos (PNM), a Política Nacional de Assistência Farmacêutica (PNAF), a Pesquisa Nacional por Amostra de Domicílios (PNAD) de 2008, do Instituto Brasileiro de Geografia e Estatísticas (IBGE) e a Pesquisa Nacional de Acesso, Utilização e Promoção do Uso Racional de Medicamentos (PNAUM).

\section{RESULTADOS}

A Política Nacional de Medicamentos (PNM), cuja finalidade é "assegurar o acesso da população a medicamentos seguros, eficazes e de qualidade, ao menor preço possível" (PNM, BRASIL, 2013), se destaca como mecanismo importante para minimizar problemas de saúde da população brasileira.

Nesse sentido, a diretriz da PNM (Relação de Medicamentos Essenciais - Rename) alcançou um patamar de visibilidade, pois o Decreto $n^{0} 7.508 / 201$ veio a regulamentar a Lei $n^{\text {o }}$ 8.080/1990, dispondo que "a Rename compreende a seleção e a padronização de medicamentos indicados para atendimento de doenças ou de agravos no âmbito do SUS" (BRASIL, 2011).

Ademais, merece realce a diretriz da Reorientação da Assistência Farmacêutica que compreende a universalização do acesso aos medicamentos no SUS por meio da descentralização do processo de dispensação de medicamentos.

Assim, houve aumento orçamentário do Componente Básico da Assistência Farmacêutica (Cbaf), representado no acesso aos medicamentos da Atenção Básica (Ação 20AE), qualificação da Assistência Farmacêutica (Ação 20AH) e apoio aos medicamentos fitoterápicos no Sistema único de Sáude (20K5) (MINISTÉRIO DA SAÚDE, BRASIL, 2018). Este aumento é observado na figura abaixo (BRASIL, 2018): 
Figura 1 - Evolução dotação do orçamento da assistência farmacêutica básica entre os anos 2009 a 2014.

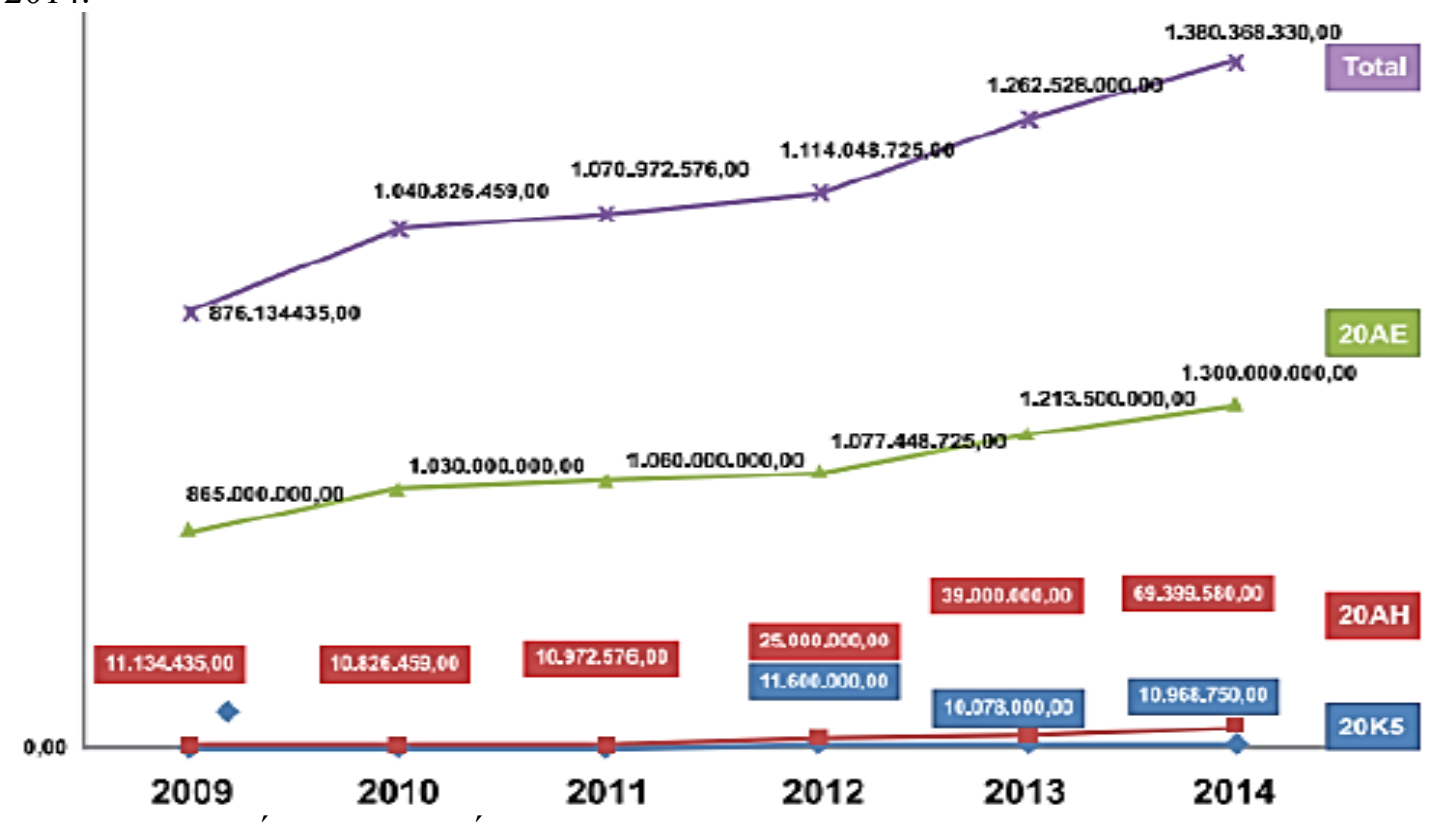

Fonte: MINISTÉRIO DA SAÚDE, BRASIL, 2018

Outrossim, o Componente Estratégico da Assistência Farmacêutica (Cesaf), desempenha papel essencial na efetivação da Política Nacional de Assistência Farmacêutica visto que sua finalidade é a garantia do acesso igualitário aos medicamentos e insumos médicos (BRASIL, 2018).

Nesse sentido, as estratégias utilizadas pelo Cesaf para a efetivação da assistência farmacêutica são: I) o monitoramento do acesso a medicamentos para doenças negligenciadas ou com pouco interesse comercial; II) a centralização da aquisição; III) produção por laboratórios públicos oficiais e; IV) a aquisições internacionais. A figura abaixo evidencia a aplicação dessas estratégias para promoção do acesso aos medicamentos. 
Figura 2 - Vias de fornecimento de medicamentos para doenças negligenciadas e relacionadas à pobreza, entre os anos 2005 a 2014 (Idem, 2018).

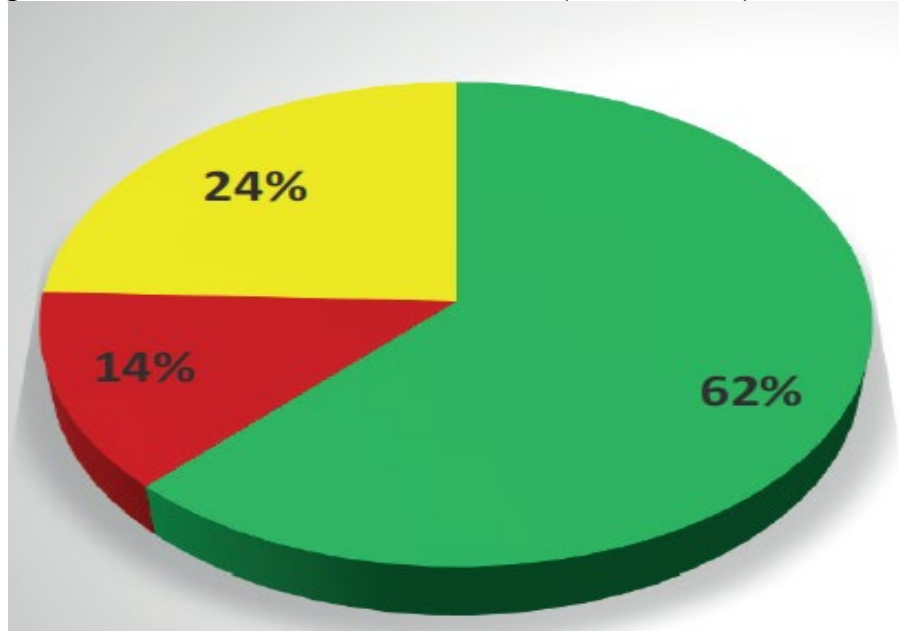

LAB. OFICIAIS

- LAB. PRIVADOS

ORG INTERNACIONAL

Fonte: Ministério da Saúde, Brasil, 2018.

Além disso, outro destaque na Promoção do Uso Racional de Medicamentos, constituída especialmente pela utilização de medidas como Campanhas Educativas, Registro e Uso de Medicamentos Genéricos, Formulário Terapêutico Nacional, Farmacoepidemiologia e Farmacovigilância e Recursos Humanos. A seguir a tabela com características demográficas do uso de medicamentos genéricos (PNAUM, 2014). 
Tabela 1 - Prevalência de uso de pelo menos um medicamento genérico segundo características demográficas e socioeconômicas.

\begin{tabular}{|c|c|c|c|}
\hline \multirow{2}{*}{ Variável } & \multicolumn{3}{|c|}{ Prevalência de uso de genéricos ${ }^{a}$} \\
\hline & $\%$ & $1 C 95 \%$ & $\mathbf{p}$ \\
\hline Sexo & & & 0,013 \\
\hline Masculino & 43,1 & $40,5-45,8$ & \\
\hline Feminino & 47,0 & $44,9-49,0$ & \\
\hline Idade (anos) & & & $<0,001$ \\
\hline $0-9$ & 33,5 & $29,1-37,8$ & \\
\hline $10-19$ & 33,0 & $26,8-39,2$ & \\
\hline $20-59$ & 43,6 & $41,5-45,7$ & \\
\hline$\geq 60$ & 56,2 & $54,1-58,3$ & \\
\hline Escolaridade ${ }^{c}$ & & & 0,819 \\
\hline $0-8$ anos & 45,5 & $43,4-47,6$ & \\
\hline 9-11 anos & 44,4 & $40,4-48,3$ & \\
\hline$\geq 12$ anos & 45,9 & $43,1-48,6$ & \\
\hline Classificação econômica ${ }^{d}$ & & & 0,040 \\
\hline $\mathrm{A} / \mathrm{B}$ & 42,6 & $39,5-45,7$ & \\
\hline $\mathrm{C}$ & 47,0 & $44,9-49,1$ & \\
\hline $\mathrm{D} / \mathrm{E}$ & 44,6 & $41,3-48,0$ & \\
\hline Região & & & $<0,001$ \\
\hline Norte & 32,7 & $28,5-37,0$ & \\
\hline Nordeste & 37,9 & $35,8-40,0$ & \\
\hline Sudeste & 49,9 & $46,8-53,0$ & \\
\hline Sul & 50,6 & $46,6-54,6$ & \\
\hline Centro-Oeste & 45,1 & $41,9-48,4$ & \\
\hline Total & 45,5 & $43,7-47,3$ & - \\
\hline
\end{tabular}

Assim, de acordo com a Pesquisa Nacional sobre Acesso, Utilização e Promoção do Uso Racional de Medicamentos (PNAUM), em se tratando de doenças crônicas, ao exemplo da hipertensão arterial, jovens adultos, de 20 a 39 anos de idade, aparecem com menor indicação médica quando o assunto é terapia medicamentosa. É o que se percebe na tabela a seguir. 


\begin{tabular}{|c|c|c|c|c|c|c|c|c|c|c|}
\hline \multirow{4}{*}{ Sexo } & \multirow{4}{*}{$\begin{array}{l}\text { Masculino } \\
\text { Feminino }\end{array}$} & \multicolumn{3}{|c|}{$\begin{array}{l}\text { Prevalência de hipertensāo } \\
\text { arterial }\end{array}$} & \multicolumn{3}{|c|}{$\begin{array}{l}\text { Pessoas hipertensas com } \\
\text { indicação médica de } \\
\text { terapia medicamentosa }\end{array}$} & \multicolumn{3}{|c|}{$\begin{array}{l}\text { Pessoas hipertensas com } \\
\text { indicaçāo médica de terapia } \\
\text { medicamentosa em uso de } \\
\text { medicamentos }\end{array}$} \\
\hline & & \multirow{3}{*}{$\begin{array}{c}\% \\
18,5 \\
28,1\end{array}$} & \multirow{3}{*}{$\begin{array}{c}\text { IC95\% } \\
{[17,4-19,8]} \\
{[27,0-29,1]}\end{array}$} & $\mathrm{p}$ & $\%$ & IC95\% & $\mathrm{p}$ & $\%$ & IC95\% & $\mathrm{p}$ \\
\hline & & & & \multirow{2}{*}{$<0,001$} & 93,3 & {$[91,4-94,8]$} & \multirow{2}{*}{0,349} & 93,4 & {$[91,5-94,9]$} & \multirow{2}{*}{0,024} \\
\hline & & & & & 94,2 & {$[92,9-95,2]$} & & 95,3 & {$[94,2-96,2]$} & \\
\hline \multirow{3}{*}{$\begin{array}{l}\text { Falxa } \\
\text { etárla }\end{array}$} & 20 a 39 anos & 5,97 & {$[5,2-6,9]$} & & 78,0 & {$[71,1-83,6]$} & & 78,4 & {$[70,8-84,5]$} & \\
\hline & 40 a 59 anos & 27,5 & {$[26,3-28,8]$} & $<0,001$ & 93,4 & {$[91,9-94,7]$} & $<0,001$ & 94,7 & {$[93,4-95,8]$} & $<0,001$ \\
\hline & Mais de 60 anos & 59,0 & {$[57,4-60,5]$} & & 98,1 & {$[97,6-98,5]$} & & 97,6 & {$[97,0-98,1]$} & \\
\hline \multirow{5}{*}{$\begin{array}{l}\text { Reglă } \\
\text { do } \\
\text { Brasll }\end{array}$} & Norte & 16,3 & {$[14,6-18,1]$} & & 92,1 & {$[89,36-94,1]$} & & 91,38 & {$[88,1-93,8]$} & \\
\hline & Nordeste & 21,7 & {$[20,3-23,1]$} & & 92,2 & {$[90,0-93,9]$} & & 91,13 & {$[87,6-93,7]$} & \\
\hline & Sudeste & 26,0 & {$[24,3-27,7]$} & $<0,001$ & 94,9 & {$[93,0-96,2]$} & 0,086 & 95,59 & {$[94,2-96,7]$} & $<0,001$ \\
\hline & Sul & 22,4 & {$[20,8-24,0]$} & & 93,7 & {$[91,0-95,6]$} & & 97,37 & {$[96,1-98,2]$} & \\
\hline & Centro-Oeste & 24,1 & {$[22,0-26,3]$} & & 92,9 & {$[90,4-94,8]$} & & 94,03 & {$[92,2-95,5]$} & \\
\hline \multirow{3}{*}{ CCEB $^{*}$} & $\mathrm{~A} / \mathrm{B}$ & 22,3 & {$[20,4-24,3]$} & & 95,1 & {$[93,0-96,6]$} & & 96,26 & {$[94,3-97,6]$} & \\
\hline & C & 23,9 & {$[22,8-25,0]$} & 0,126 & 93,4 & {$[91,9-94,7]$} & 0,302 & 94,78 & {$[93,6-95,8]$} & 0,017 \\
\hline & $\mathrm{D} / \mathrm{E}$ & 24,7 & {$[23,1-26,3]$} & & 93,6 & {$[91,5-95,2]$} & & 92,31 & {$[89,2-94,6]$} & \\
\hline Total & & 23,7 & {$[22,8-24,6]$} & & 93,8 & {$[92,8-94,8]$} & & 94,6 & {$[93,5-95,5]$} & \\
\hline
\end{tabular}

Fonte: Pnaum, Brasil, 2014

O Programa Farmácia Popular do Brasil (PFPB) criado em 2004 pelo Decreto n $^{\text {o }}$ 5.090, que regulamentou a Lei 10.858 de 2004, veio para fortalecer a Política Nacional de Assistência Farmacêutica (PNAF), pois em 2006 após a publicação da Portaria $n^{0}$ 491, que autorizou o governo federal a formar parcerias com o setor farmacêutico privado, instituiu a ação "aqui tem farmácia popular".

Logo, o PFPB já atendeu cerca de 47.416.735 de usuários até o final de 2017, número que representa aproximadamente $23 \%$ da população brasileira que teve acesso aos medicamentos por consequência da implementação desse programa. Em termos financeiros, os custos para o Ministério da Saúde com o PFPB, em 2006 chegaram ao montante de R\$ 34.723.571, passando para $\mathrm{R} \$ 2.815 .000 .000,00 \mathrm{em} 2017$.

\section{DISCUSSÃO}

A concretização do direito à saúde, compreendendo os medicamentos deve ser amparada por um sistema de saúde eficiente, como mecanismo de concretização de direitos e garantias fundamentais, ao exemplo, da dignidade da pessoa humana. Neste sentido, o artigo 12, do Pacto Internacional sobre Direitos Econômicos, Sociais e Culturais (PIDESC), onde 
“os Estados Partes do presente Pacto reconhecem o direito de toda pessoa de desfrutar o mais elevado nível possível de saúde física e mental" (BRASIL, 1992).

O direito fundamental à saúde está consagrado no artigo $6^{\circ}$ da Constituição Federal de 1988 como um direito social. Saúde em sentido amplo pode ser conceituada como um estado de harmonia funcional entre físico e psíquico de uma pessoa. $\mathrm{O}$ direito à saúde é visualizado sob dois panoramas: um objetivo que protege o titular contra as arbitrariedades do Estado e dos particulares; e outro prestacional que efetiva o acesso à saúde (BATISTA et al., 2015).

$\mathrm{O}$ direito à saúde pode ser efetivado mediante políticas públicas ${ }^{2}$ sociais e econômicas. Por este motivo, o direito à saúde, especificamente aos medicamentos, pode e deve ser garantido por meio de políticas públicas de assistência farmacêutica, que tratam da "proteção e recuperação da saúde individual e coletiva, tendo os medicamentos como insumos essenciais e visando à viabilização do acesso aos mesmos" (XAVIER et al, 2017).

Apesar do advento da Lei $\mathrm{n}^{\circ}$ 8.080/1990 dando origem ao SUS, em clara convergência com a Constituição Federal de 1988, a Política Nacional de Medicamentos ainda não alcançou sua plenitude, pois:

\footnotetext{
Ainda que as diretrizes e as prioridades desta política sejam consideradas adequadas, há desafios e fragilidades na implementação de qualquer política pública. Destacamse a trajetória de subfinanciamento com a qual historicamente convivem as ações e os serviços públicos de saúde desde o advento do SUS e o desequilíbrio entre o gasto público e o privado com medicamentos (Ibid., 2017, p. 2609).
}

Logo, a literatura nacional ensina que os medicamentos são insumos que desempenham papel fundamental no sistema de saúde, contribuindo na melhoria da qualidade de vida das populações. Assim, sobre o medicamento genérico, é possível afirmar que se traduziu em uma agenda do poder público para a promoção do acesso aos medicamentos essenciais (MIRANDA et. al., 2009, p. 2147-48).

Portanto, a Política Nacional de Medicamentos (PNM) é componente importante da Política Nacional de Saúde (PNS), e visa promover melhorias nas ações de assistência à saúde. Para avaliar esta política foi preciso incluir estudos que analisam a eficiência e alcance da promoção ao acesso a medicamentos e custos para o poder público. Neste estudo, o sujeito de análise consistiu em demonstrar a convergência da Política Nacional de Medicamentos com as diretrizes de Direitos Humanos.

\footnotetext{
${ }^{2}$ Políticas públicas também abarcam uma série de questões setoriais que são entrelaçadas, assíncronas e espacialmente sobrepostas (FURTADO et al., 2015).
}

PIDCC, Aracaju/Se, Ano VIII, Volume 13 n 03, p.093 a 105 Out/2019 | www.pidcc.com.br 


\section{CONCLUSÃO}

O estudo da Política Nacional de Medicamentos (PNM) permitiu avaliar a conformidade entre suas ações e os Direitos Humanos. Com isto, os resultados obtidos, bem como a discussão apontaram para a existência de simetria entre a PNM e as diretrizes de Direitos Humanos relacionadas à saúde, promovendo o acesso aos medicamentos.

A PNM implementou um modelo de assistência farmacêutica básica, e conforme dito em tópicos anteriores, a descentralização das ações de assistência farmacêutica ganharam destaque, não somente pelo atendimento às necessidades locais, mas por fomentar que Estados e Municípios cumprissem metas pré-estabelecidas.

Conclui-se portanto, que a Política Nacional de Medicamentos (PNM) vem ao longo dos anos, melhorando o acesso aos medicamentos, uma vez que a cada dois anos, esta relação é atualizada, alinhando-se com as diretrizes da Organização Mundial da Saúde (OMS) sobre medicamentos essenciais, em sintonia com os Direitos Humanos.

\section{REFERÊNCIAS}

BRASIL. Constituição da República Federativa do Brasil (1988). In: ANGHER, Anne Joyce (org.). Vade Mecum acadêmico de direito Rideel. 24. ed. São Paulo: Rideel, 2017.

BRASIL. Portaria no 3.916, de 30 de outubro de 1988. Disponível em:http://bvsms.saude.gov.br/bvs/saudelegis/gm/1998/prt3916_30_10_1998.html. Acesso em: 28 jun. 2019.

BRASIL. Resolução no 338, de 06 de maio de 2004. Disponível em: http://bvsms.saude.gov.br/bvs/saudelegis/cns/2004/res0338_06_05_2004.html. Acesso em: 28 jun. 2019.

BRASIL. Pesquisa Nacional por Amostra de Domicílios (PNAD). Instituto Brasileiro de Geografia e Estatísticas, 2008. Disponível em: https://biblioteca.ibge.gov.br/pt/bibliotecacatalogo?view=detalhes\&id=236151. Acesso em 29 jun. 2019. 
BRASIL. Lei $\mathbf{n}^{\mathbf{0}} \mathbf{1 0 . 8 5 8}$, de 13 de abril de 2004. Disponível em: http://www.planalto.gov.br/ccivil_03/_ato2004-2006/2004/lei/110.858.htm. Acesso em: 30 jun. 2019.

BRASIL. Decreto $\mathbf{n}^{0}$ 5.090, de 20 de maio de 2004. Disponível em: http://www.planalto.gov.br/ccivil_03/_ato2004-2006/2004/decreto/d5090.htm. Acesso em: 30 jun. 2019.

BRASIL. Lei $\mathbf{n}^{\mathbf{0}}$ 8.080, de 19 de setembro de 1990. Disponível em: http://www.planalto.gov.br/ccivil_03/leis/18080.htm. Acesso em: 28 jun. 2019.

BRASIL. Decreto no 793, de 5 de abril de 1993. Disponível em: http://www.planalto.gov.br/ccivil_03/decreto/1990-1994/D0793.htm. Acesso em: 30 jun. 2019.

BRASIL. Decreto $\mathbf{n}^{0}$ 3.181, de 23 de setembro de 1999. Disponível em:

http://www.planalto.gov.br/ccivil_03/decreto/D3181.htm\#art10. Acesso em: 30 jun. 2019.

BRASIL. Lei n 9.787, de 10 de fevereiro de 1999. Disponível em: http://www.planalto.gov.br/ccivil_03/LEIS/L9787.htm. Acesso em: 29 jun. 2019.

BRASIL. Lei $\mathbf{n}^{\mathbf{0}}$ 6.360, de 23 de setembro de 1976. Disponível em:

http://www.planalto.gov.br/ccivil_03/LEIS/L6360.htm. Acesso em: 29 jun. 2019.

BRASIL. Pesquisa Nacional de Saúde (2013). Disponível em:

http://portalarquivos2.saude.gov.br/images/pdf/2014/dezembro/18/PNS-2013.pdf. Acesso em: 29 jun. 2019.

BRASIL. Ministério da Saúde. Secretaria de Ciência, Tecnologia e Insumos Estratégicos. Departamento de Assistência Farmacêutica e Insumos Estratégicos. Relação Nacional de Medicamentos Essenciais: RENAME 2018. Brasília: Ministério da Saúde, 2018. Disponível em: http://portalms.saude.gov.br/assistencia-farmaceutica/medicamentos-rename.

BRASIL. Assistência farmacêutica no SUS. Conselho Nacional de Secretários de Saúde. Brasília: CONASS, 2007.

BRASIL. Ministério da Saúde. Secretaria de Ciência, Tecnologia e Insumos Estratégicos. Assistência farmacêutica no SUS: 20 anos de políticas e propostas para desenvolvimento e PIDCC, Aracaju/Se, Ano VIII, Volume 13 no 03, p.093 a 105 Out/2019 | www.pidcc.com.br 
qualificação: relatório com análise e recomendações de gestores, especialistas e representantes da sociedade civil organizada. Brasília: Ministério da Saúde,2018. Disponível em:

http://bvsms.saude.gov.br/publicacoes/assistencia_farmaceutica_sus_relatorio_recomendacoe s.pdf.

BRASIL. Pacto Internacional sobre Direitos Econômicos, Sociais e Culturais. Decreto $\mathbf{n}^{\mathbf{0}}$ 591, de 6 de julho de 1992. Disponível em: http://www.planalto.gov.br/ccivil_03/decreto/1990-1994/d0591.htm. Acesso em: 30 jun. 2019.

BRASIL. Ministério da Saúde. Secretaria de Ciência, Tecnologia e Insumos Estratégicos. Departamento de Assistência Farmacêutica e Insumos Estratégicos. Coordenação Geral do Componente Especializado da Assistência Farmacêutica. Componente Especializado da Assistência Farmacêutica: inovação para a garantia do acesso a medicamentos no SUS. Brasília: Ministério da Saúde, 2014.

BERTOLDI, A.D et al. Utilização de medicamentos genéricos na população brasileira: uma avaliação da PNAUM 2014. Revista de Saúde Pública. São Paulo, v. 50. supl.2. 2016. Disponível em: http://www.scielo.br/scielo.php?script=sci_arttext\&pid=S003489102016000300309. Acesso em: 29 jun. 2019.

BATISTA, C. K; CALIL, M. L. O direito fundamental de acesso a medicamentos e a função social da propriedade imaterial no brasil. Revista De Direito Sanitário, v. 17. n. 1. p. 106121. 2016. Disponível em: https://doi.org/10.11606/issn.2316-9044.v17i1p106-121. Acesso em: 30 jun.2019.

COMISSÃO NACIONAL DE INCORPORAÇÃO DE TECNOLOGIAS NO SUS. Relação nacional de medicamentos essenciais de 2018. Disponível em: http://conitec.gov.br/relacaonacional-de-medicamentos-essenciais-de-2018-e-publicada. Acesso em: 30 jun. 2019.

FURTADO, Bernardo Alves; SAKOWSKI, Patrícia; TÓVOLLI, Marina Eds. Modelagem de sistemas complexos para políticas públicas. IPEA, Brasília, 2015.

MIRANDA, Elaine Silva et al. Disponibilidade no setor público e preços no setor privado: um perfil de medicamentos genéricos em diferentes regiões do Brasil. Revista Cadernos de Saúde Pública. Rio de Janeiro, v. 25. n. 10. p. 2147-2158. Disponível em: https://scielosp.org/pdf/csp/2009.v25n10/2147-2158/pt. Acesso em: 30 jun.2019.

ORGANIZAÇÃO MUNDIAL DA SAÚDE. ORGANIZAÇÃO PAN-AMERICANA DA SAÚDE. Lista de medicamentos essenciais. 2017. Disponível em: https://apps.who.int/iris/bitstream/handle/10665/273826/EML-20-eng.pdf?ua=1. Acesso em: 30 jun. 2019. 
Política Nacional de Medicamentos. Informes Técnicos Institucionais. Secretaria de Políticas de Saúde. Revista de Saúde Pública. São Paulo, v. 34. n. 2. p. 206-9. Disponível em: http://www.scielo.br/scielo.php?script=sci_arttext\&pid=S0034-89102000000200018. Acesso em: 30 jun. 2019.

VASCONCELOS, Daniela Moulin Maciel et al. Política nacional de medicamentos em retrospectiva: um balanço de (quase) 20 anos de implementação. Revista Ciência \& Saúde Coletiva. Rio de Janeiro, v. 22. n. 8. p. 2609-14. Disponível em:

http://www.scielo.br/scielo.php?script=sci_arttext\&pid=S1413-81232017002802609. Acesso em: 30 jun. 2019.

XAVIER, Elton Dias; FERREIRA, Sheile Nayara. O licenciamento compulsório de patente de medicamentos. $3^{\circ}$ Encontro Internacional de Pesquisadores em Esporte, Saúde, Psicologia e Bem-Estar (EIPSE). Revista portuguesa de ciências do desporto. Porto. 2016. Disponível em: https://rpcd.fade.up.pt/_arquivo/artigos_soltos/2017-S2A/03.pdf. Acesso em: 24 abr. 2019. 